\title{
Stereospecific $\operatorname{Pd}(0)$-Catalyzed Arylation of an
}

\section{Allylic Hydroxy Phosphonate Derivative: Formal \\ Synthesis of $(\mathrm{S})(+)$-ar-Turmerone}

\author{
Bradley J. Rowe and Christopher D. Spilling* \\ Department of Chemistry and Biochemistry, University of Missouri-St. Louis, \\ 8001 Natural Bridge Road, St. Louis, Missouri 63121. \\ cspill@umsl.edu
}

\section{Supporting Information}

Table of Contents

General Experimental

S2

Stereochemical Analysis

${ }^{1} \mathrm{H}$ NMR spectrum for $\mathbf{3}$

S4

${ }^{13} \mathrm{C}$ NMR spectrum for 3

S5

${ }^{1} \mathrm{H}$ NMR spectrum for $\mathbf{6 E}$

S6

${ }^{1} \mathrm{H}\left\{{ }^{31} \mathrm{P}\right\}$ NMR Spectrum for $\mathbf{6 E}$

S7

${ }^{13} \mathrm{C}$ NMR spectrum for $\mathbf{6 E}$

S8

${ }^{1} \mathrm{H}$ NMR spectrum for $\mathbf{6 Z}$

S9

${ }^{1} \mathrm{H}\left\{{ }^{31} \mathrm{P}\right\}$ NMR spectrum for $\mathbf{6 Z}$ 
${ }^{13} \mathrm{C}$ NMR spectrum for $\mathbf{6 Z}$

${ }^{1} \mathrm{H}$ NMR spectrum for 7

S12

${ }^{13} \mathrm{C}$ NMR spectrum for 7

S13

${ }^{31} \mathrm{P}$ NMR spectrum for 8

S14

${ }^{1} \mathrm{H}$ NMR spectrum for 8

S15

${ }^{13} \mathrm{C}$ NMR spectrum for 8

S16

A comparison of HPLC data for compound 7

prepared from $( \pm) 6,(3 S, 1 E) 6$ and $(3 R, 1 Z) 6$

General Experimental: ${ }^{1} \mathrm{H},{ }^{13} \mathrm{C}$ and ${ }^{31} \mathrm{P}$ NMR spectra were recorded at 300,75 and $121 \mathrm{MHz}$, repectively in $\mathrm{CDCl}_{3}$. ${ }^{1} \mathrm{H} \mathrm{NMR}$ spectra are referenced to internal tetramethylsilane (TMS, $\delta=$ $0.00),{ }^{13} \mathrm{C}$ NMR spectra to the center-line of $\mathrm{CDCl}_{3}(77.23 \mathrm{ppm})$ and ${ }^{31} \mathrm{P} \mathrm{NMR}$ spectra to external $85 \% \mathrm{H}_{3} \mathrm{PO}_{4}$. Coupling constants, $J$, are reported in $\mathrm{Hz}$. Enantiomer ratios were measured by chiral stationary-phase HPLC on a $(S, S)$-Whelk-O 1 column or a Chiralpak AS column. Optical rotations were determined using a polarimeter set at $589 \mathrm{~nm}$. (1R) (2E) dimethyl (1-hydroxy-2butenyl) phosphonate was prepared using published procedures. ${ }^{1,2} \quad$ (1S,2E) Dimethyl (1hydroxy-2-butenyl) phosphonate (1) was prepared according to published procedures using Ddimethyl tartrate as the ligand and the lipase from rhizopus arrhizus (Sigma) $\left(2.6 \mathrm{~g}, 86 \%\right.$ e.e.). ${ }^{2}$

1. a) Texier-Boullet, F.; Foucaud, A. Synthesis 1982, 165; b) Baraldi, P. G.; Guarneri, M.; Moroder, F.; Pollini, G. P.; Simoni, D. Synthesis 1982, 653.

2. Rowe, B.J.; Spilling, C. D. Tetrahedron Asymm. 2001, 12, 1701 
Stereochemical Analysis For the (R) Hydroxy Phosphonate (65\% e.e.)



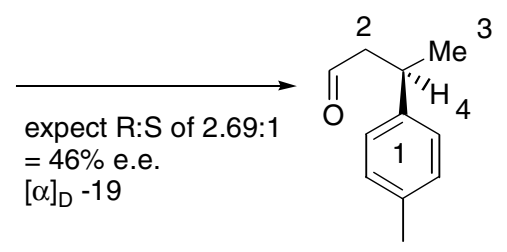

$\mathrm{R}$ isomer

$[\alpha]_{D}-15.2(=37 \%$ e.e $)$

a $43 \%$ total erosion in e.e.

R S

$4.8: 1$



$\begin{array}{rr}4.08 & 0.85 \\ +0.15 & +0.72\end{array}$

$4.23: 1.57=2.69: 1=46 \%$

\section{Stereochemical Analysis For the (S) Hydroxy Phosphonate (86\% e.e.)}

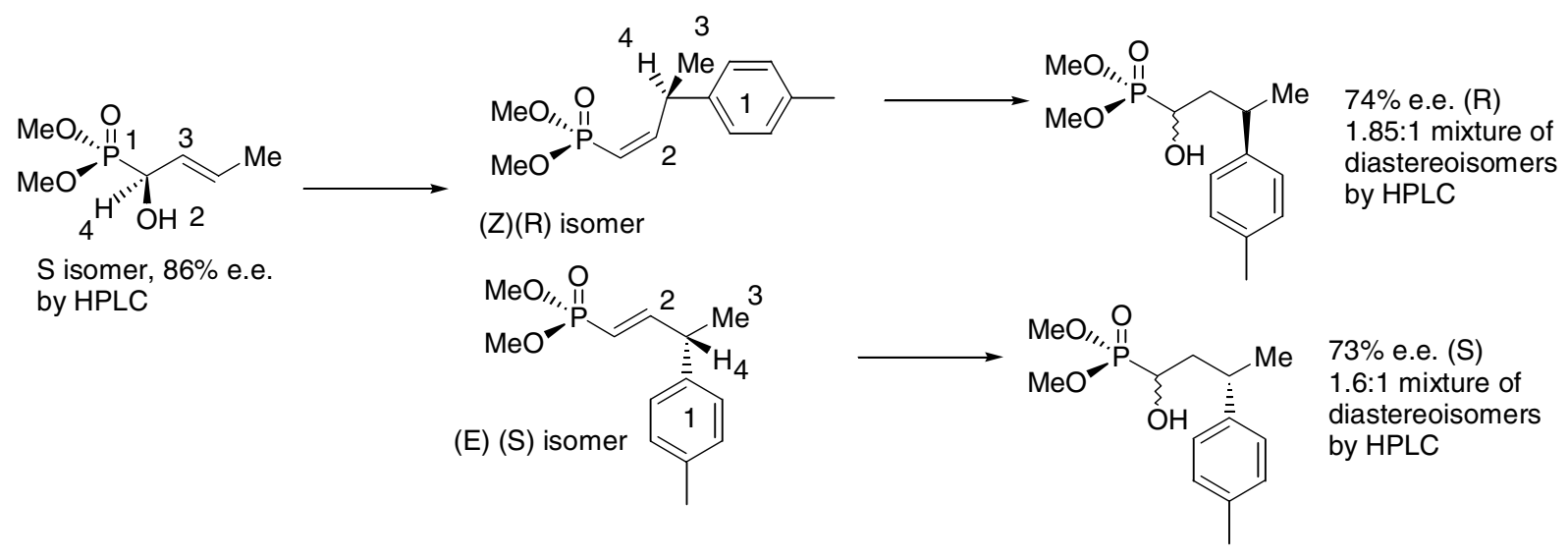

(S) isomer

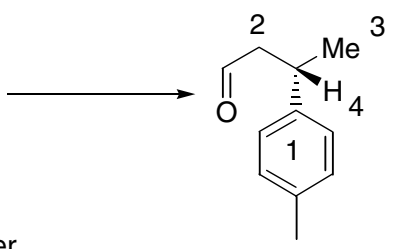

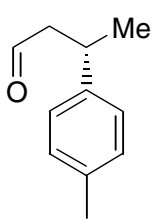

(S)-(+)-3-(p-tolyl)-butanal

$95.5 \%$ e.e. $[\alpha]_{D}+39.6\left(\mathrm{c} 1, \mathrm{CHCl}_{3}\right)$ pure $=[\alpha]_{D}+41.5$

\section{Accounting for $E$ and $Z$ isomer formation, expected e.e. $+46 \%$}

$37 \%$ represents a $14 \%$ erosion in e.e. 


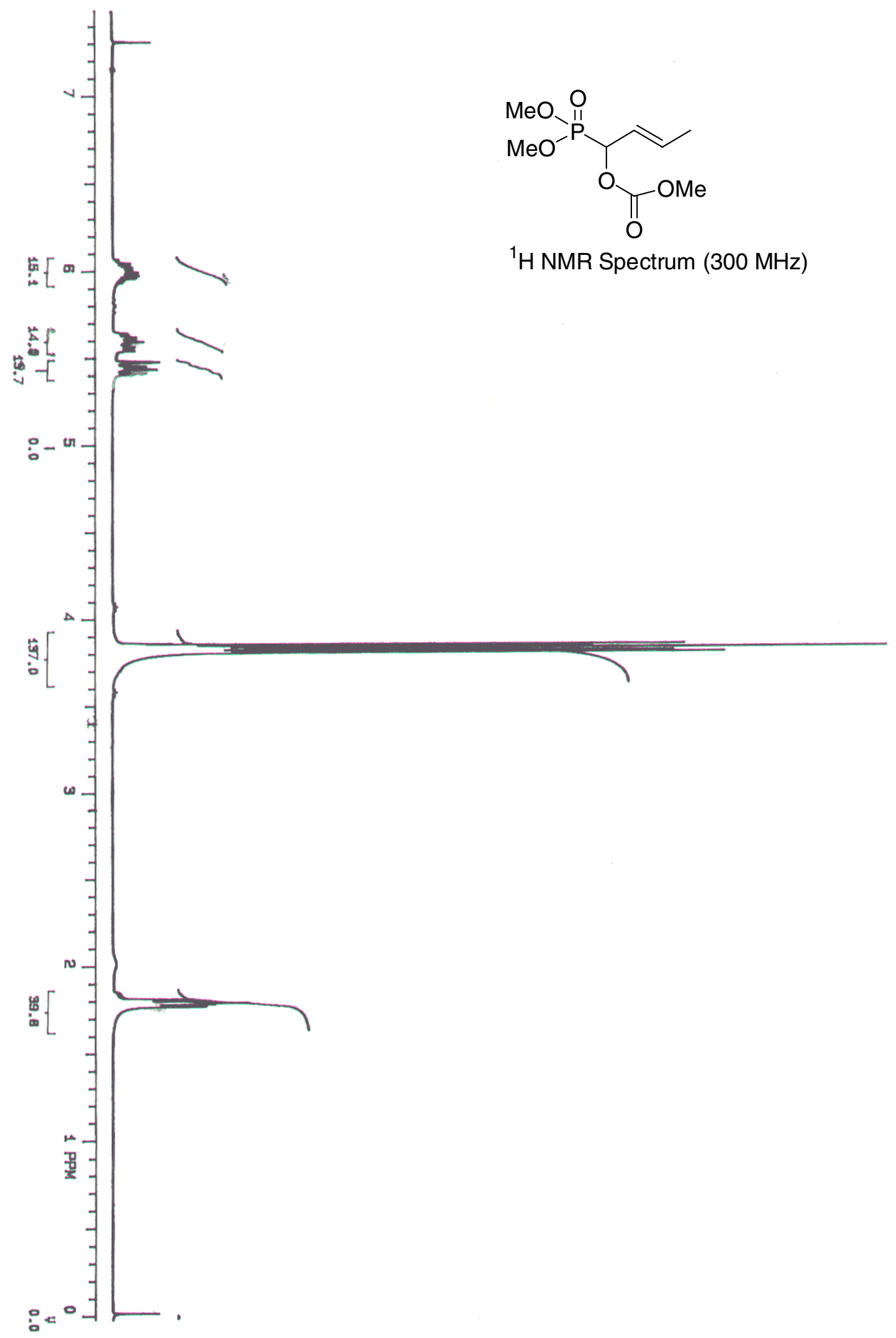




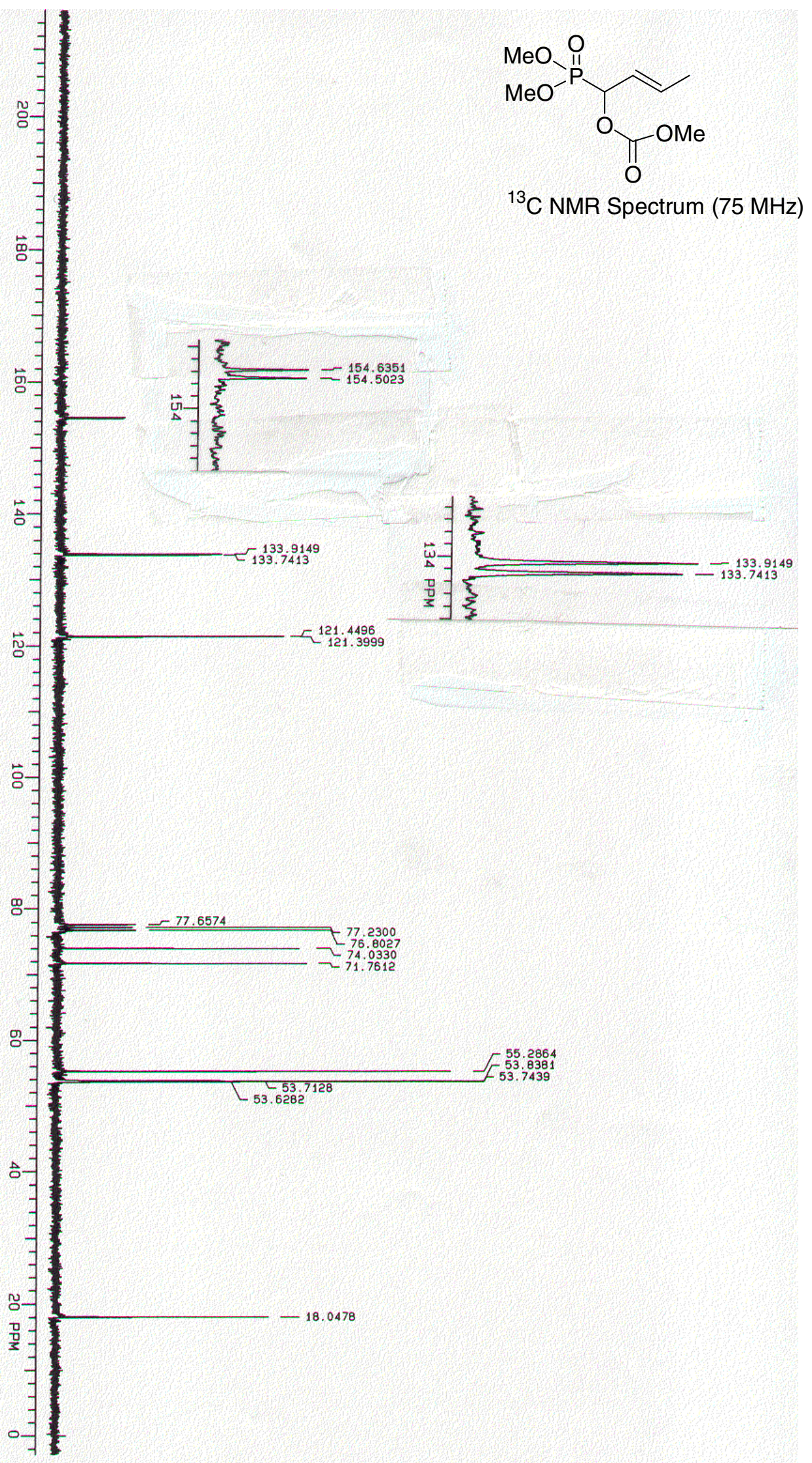




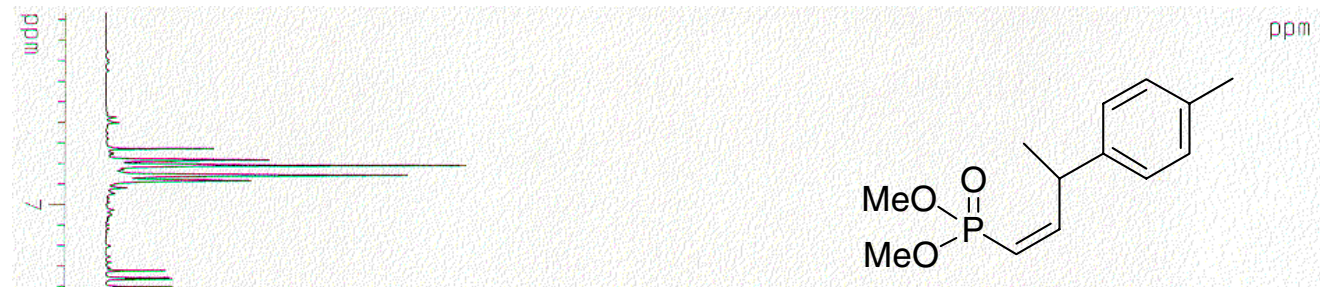

${ }^{1} \mathrm{H}$ NMR Spectrum $(300 \mathrm{MHz})$

on
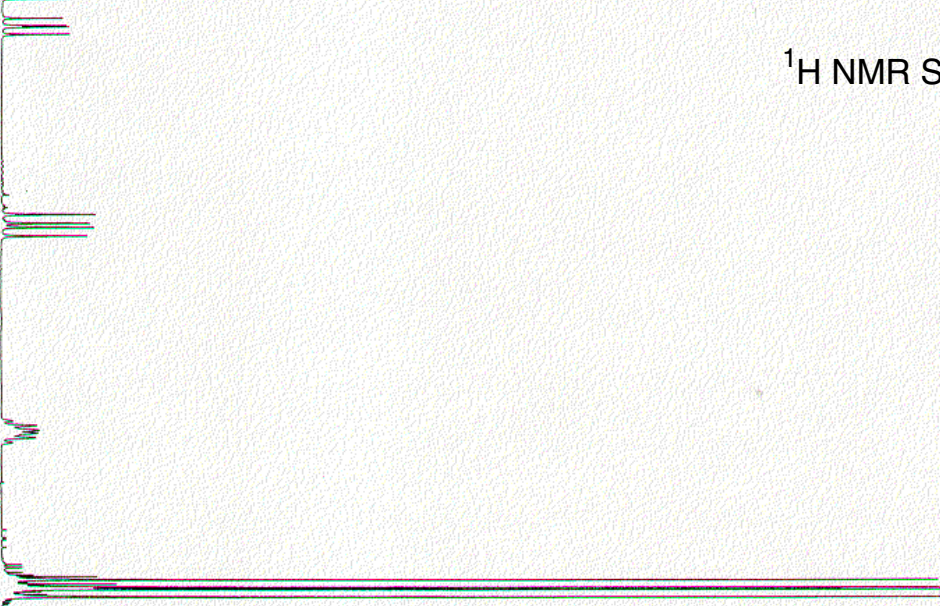

$=$



$\begin{array}{r}-3.76137 \\ -\quad 3.72420 \\ \hline\end{array}$

3.72420
-3.71452

$-3.67719$

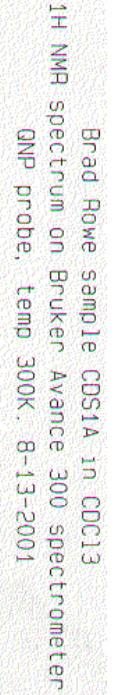

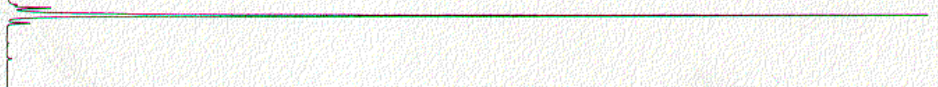

$-2.32113$
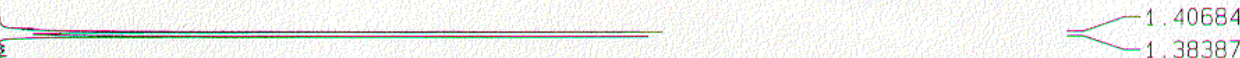

$-0.00586$

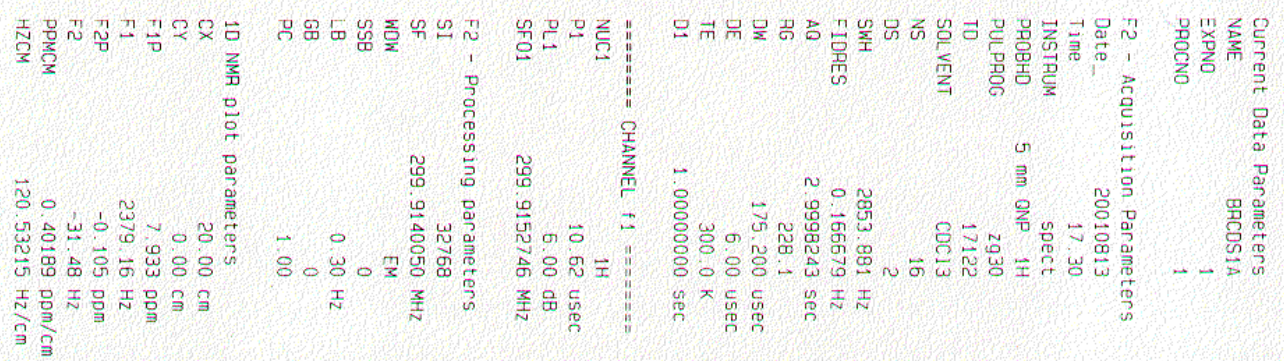




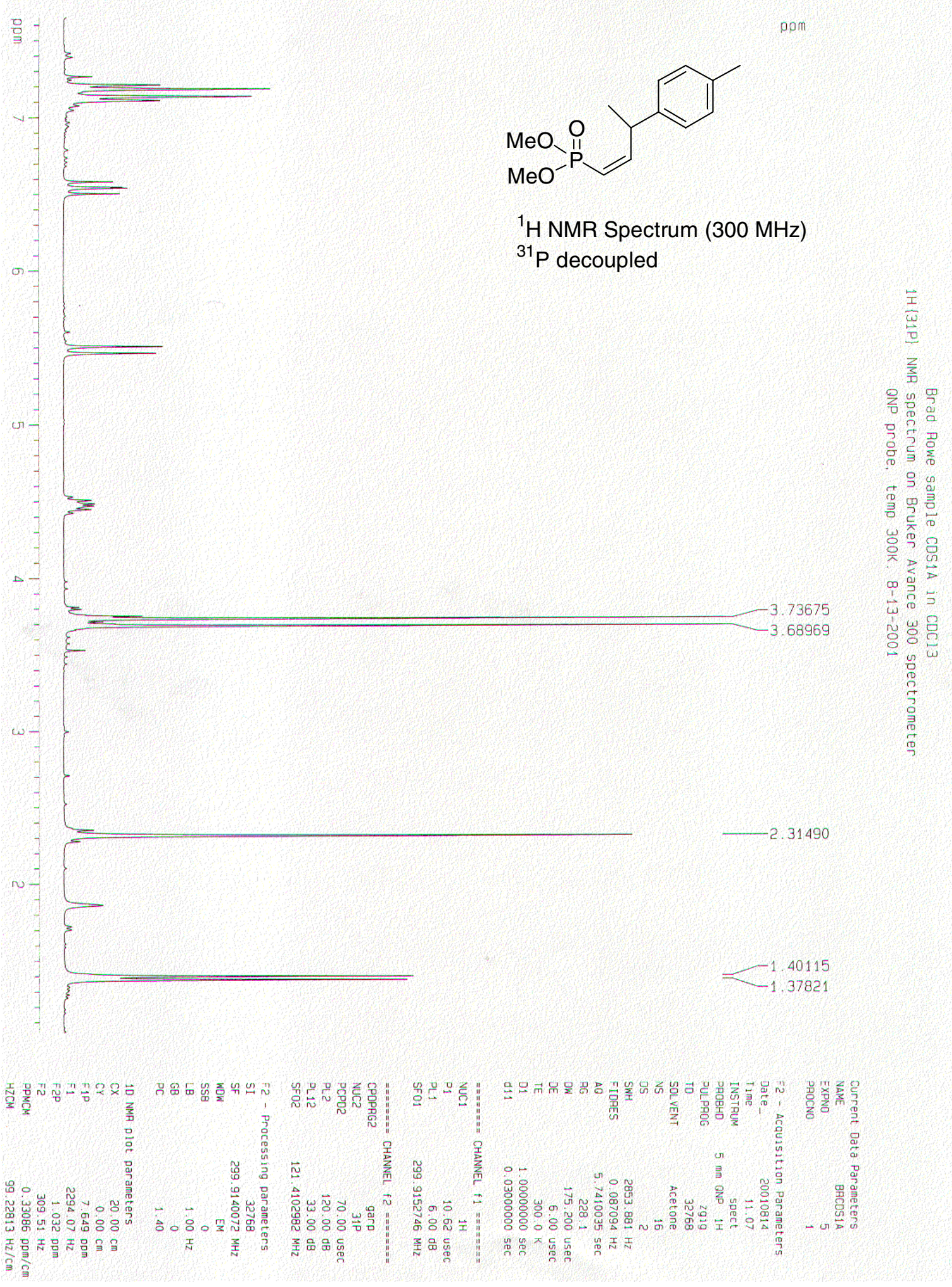









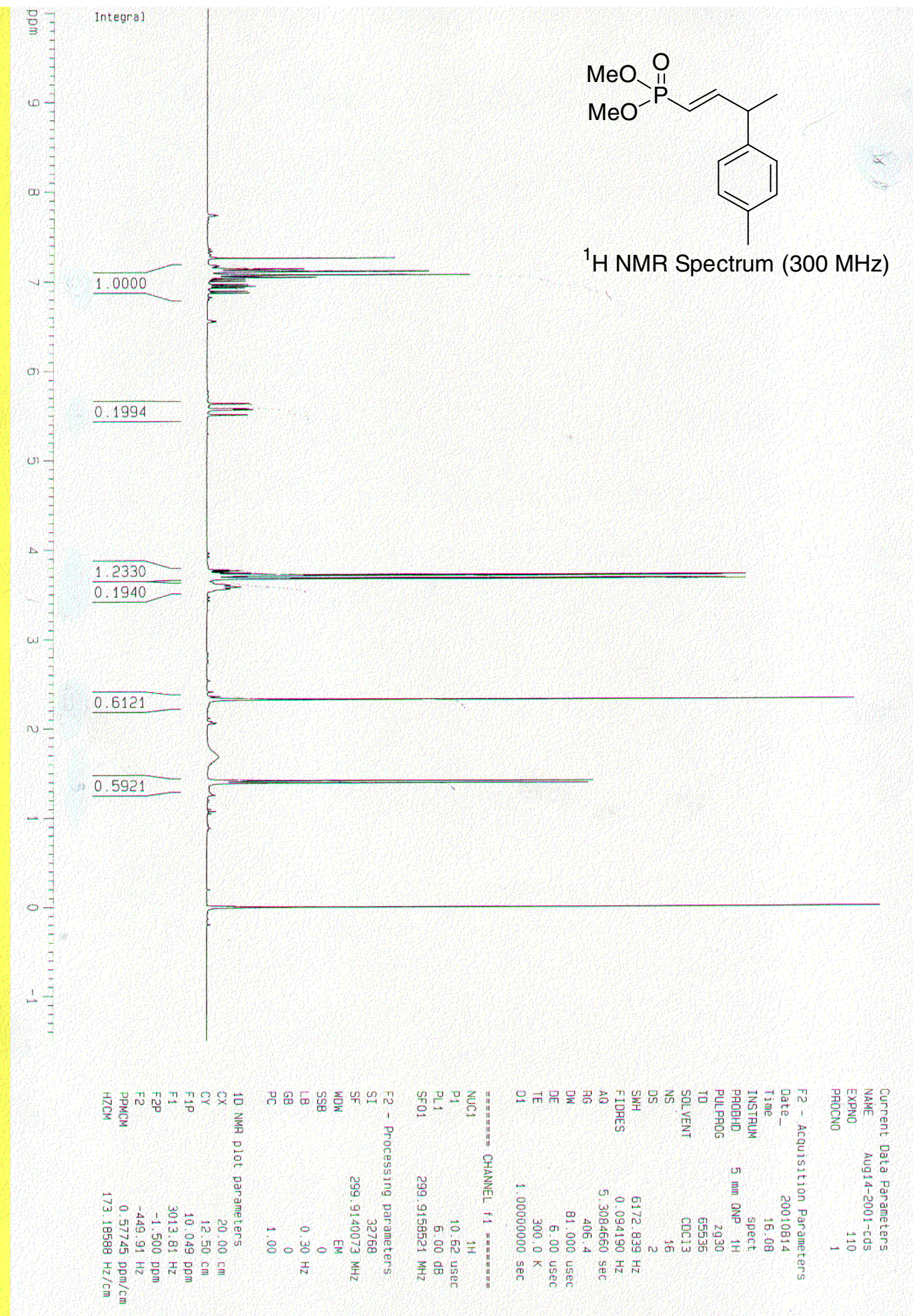









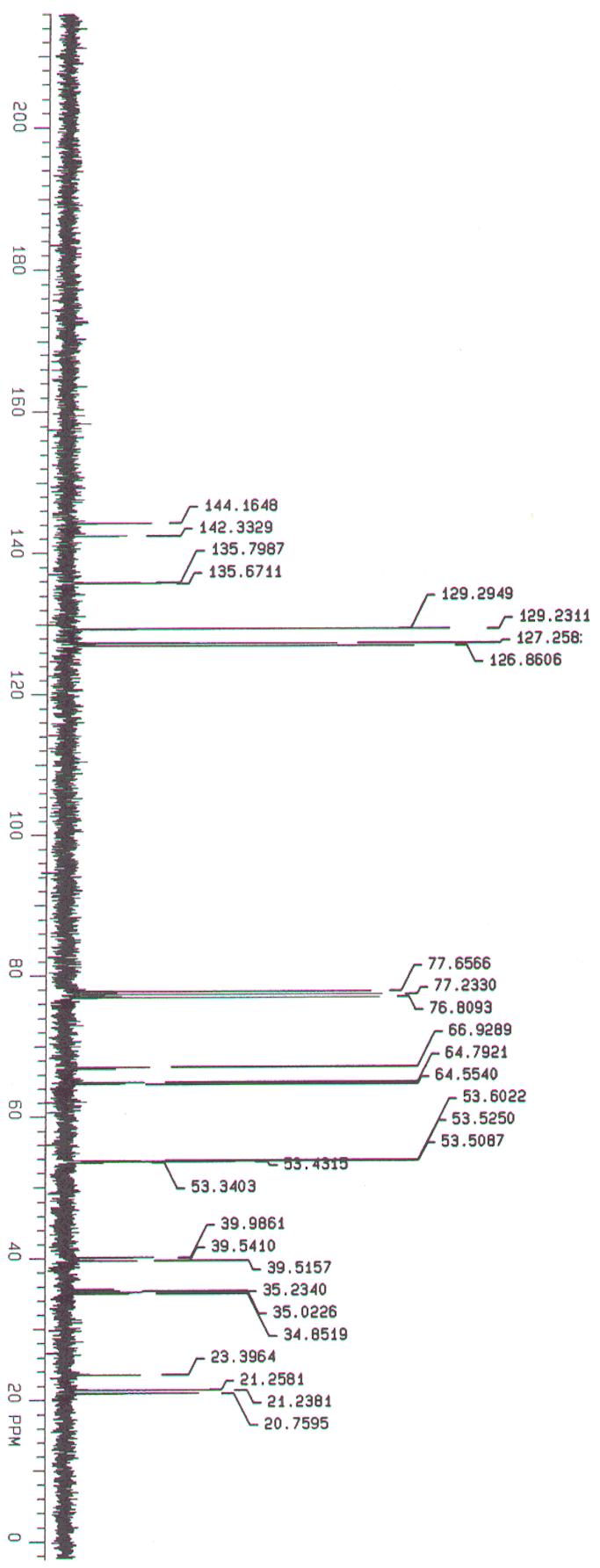





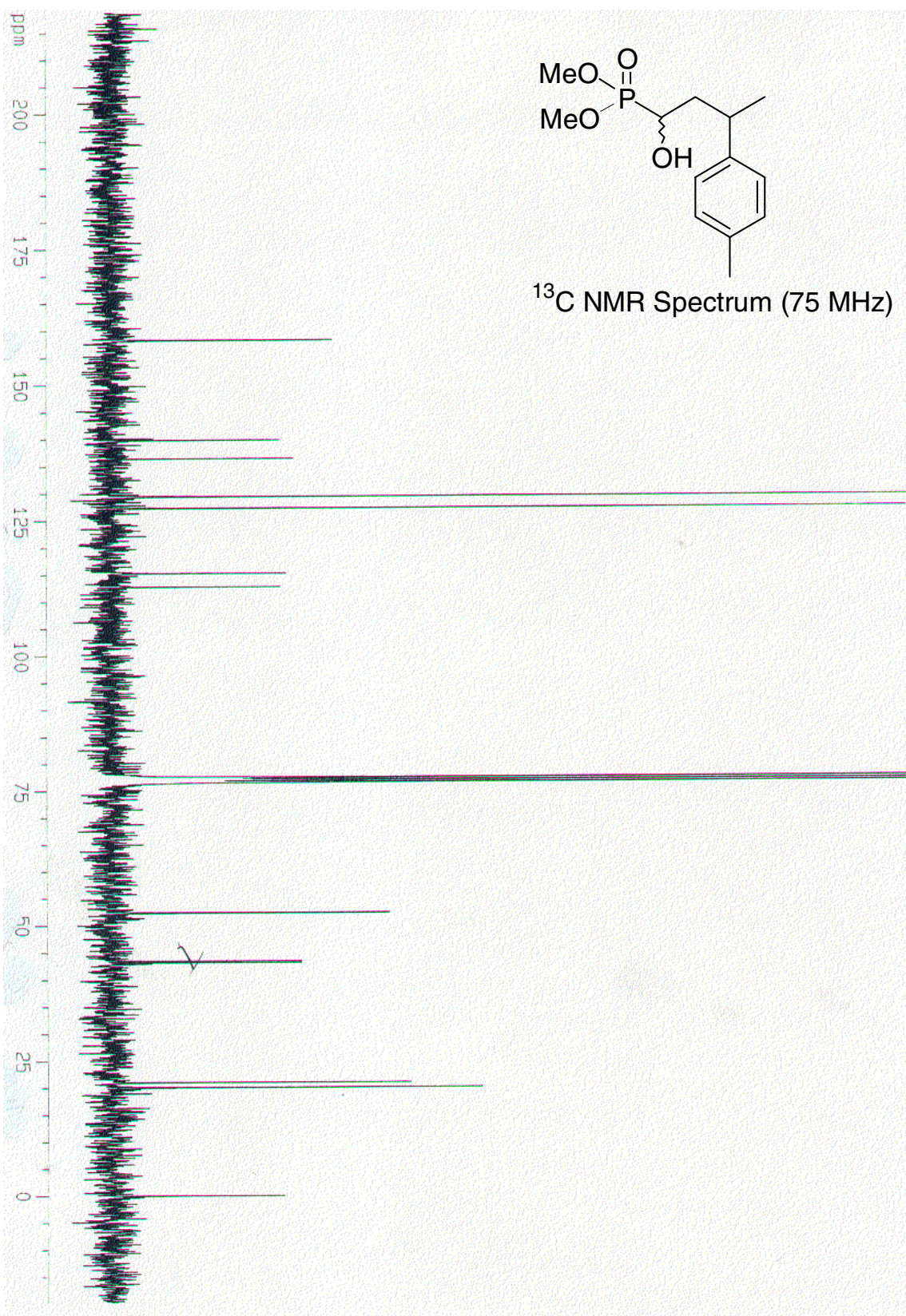

\section{NMR Spectrum (75 MHz)}

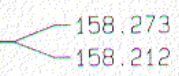

$-139.828$

$-136.430$

$-129.406$

$-115.237$

$-112.738$

77.472

$-77.048$

$-76.624$

$-52.380$

$\begin{array}{r}-52.306 \\ \hline\end{array}$

43.455
+

$-43.173$

-21.016
$-\quad 20.081$

0.011
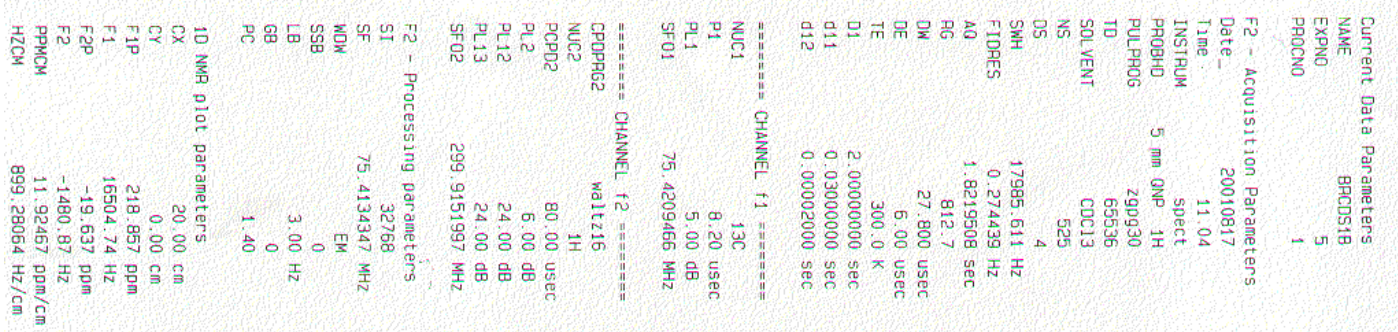


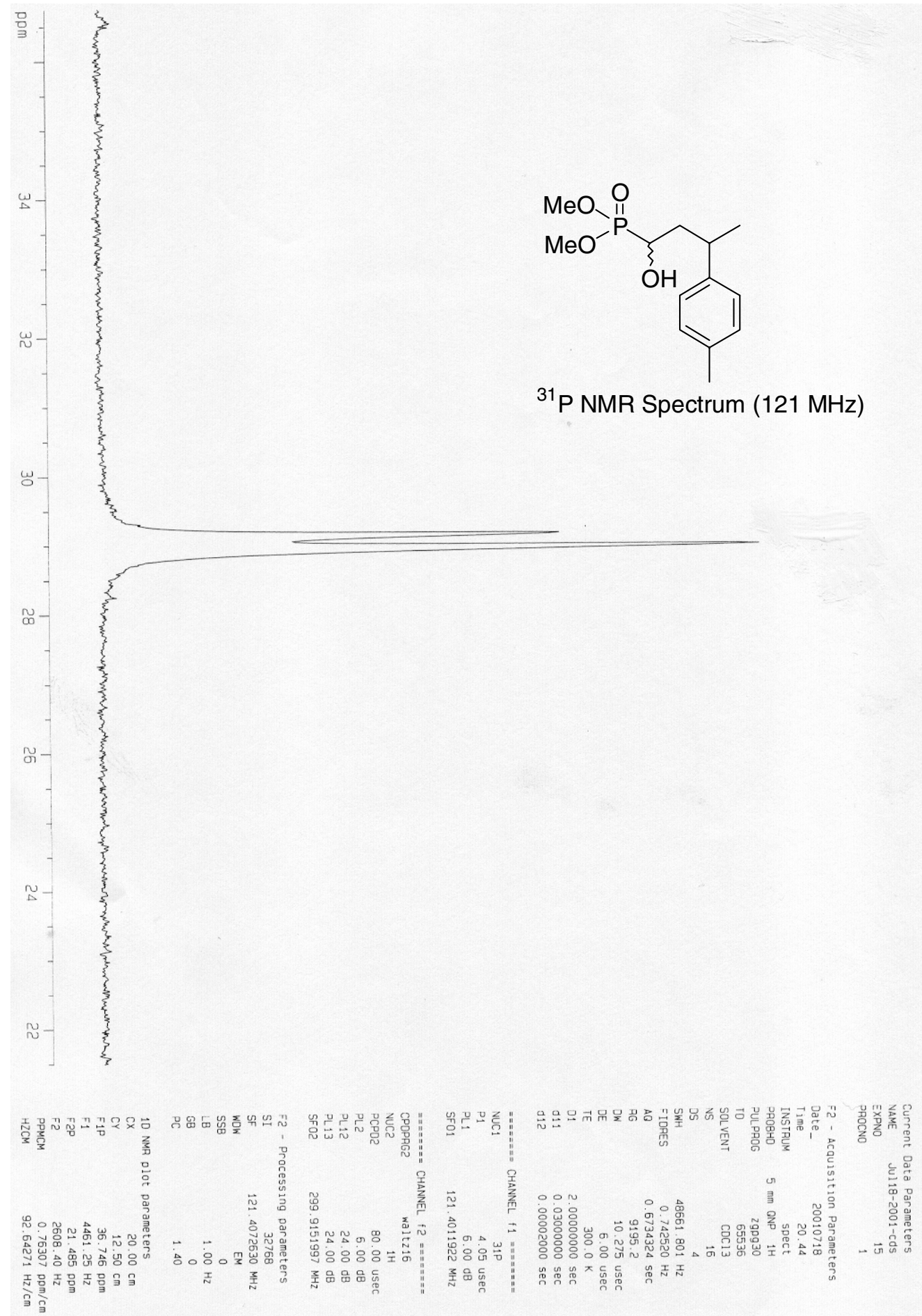








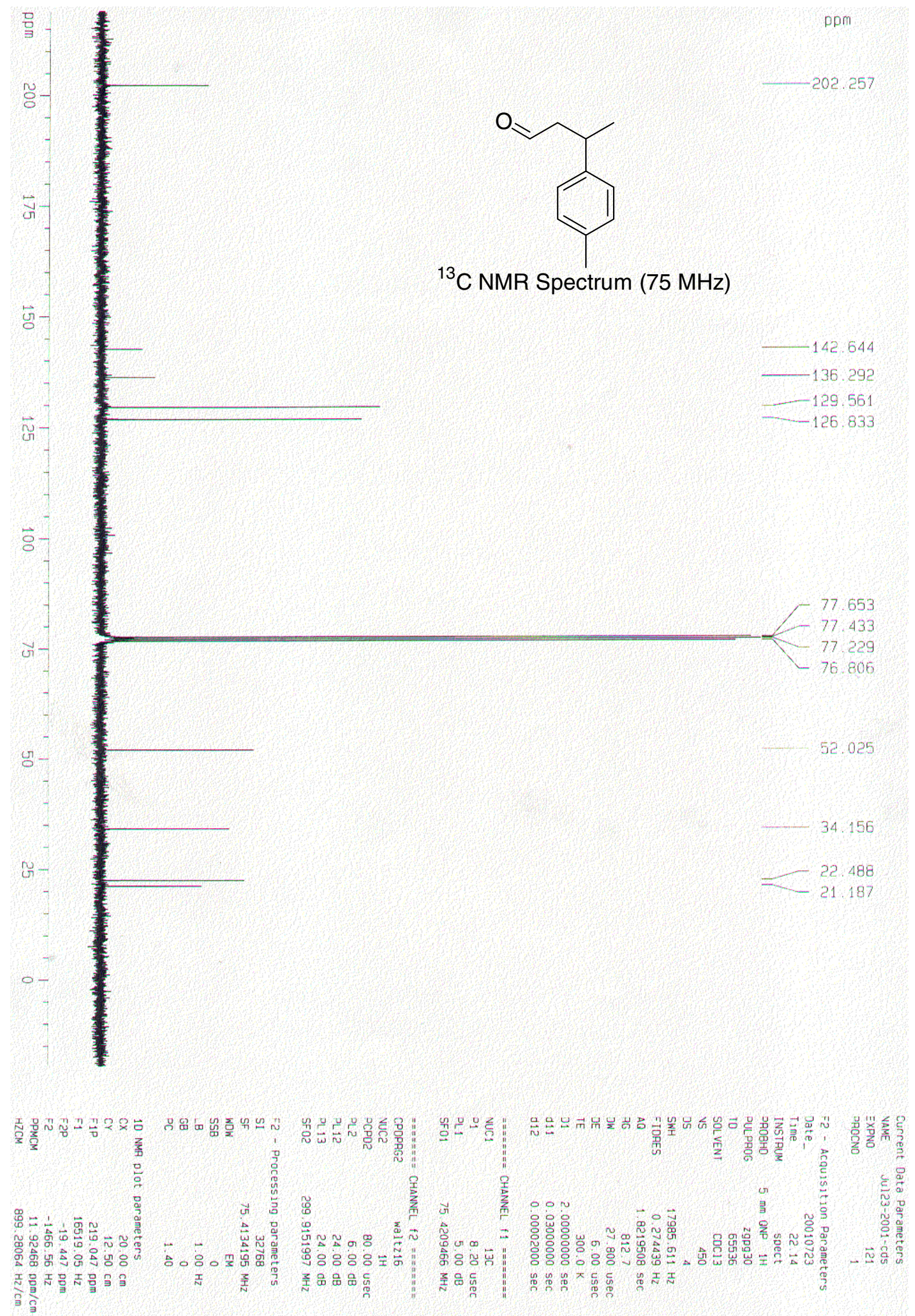


\section{HPLC Data:}

Dual column system: WhelkO S,S column followed by a ChiralPak AS column, detection at $205 \mathrm{~nm}, 95: 5 \mathrm{EtOH}$ hexane, $1 \mathrm{~mL}$ per minute
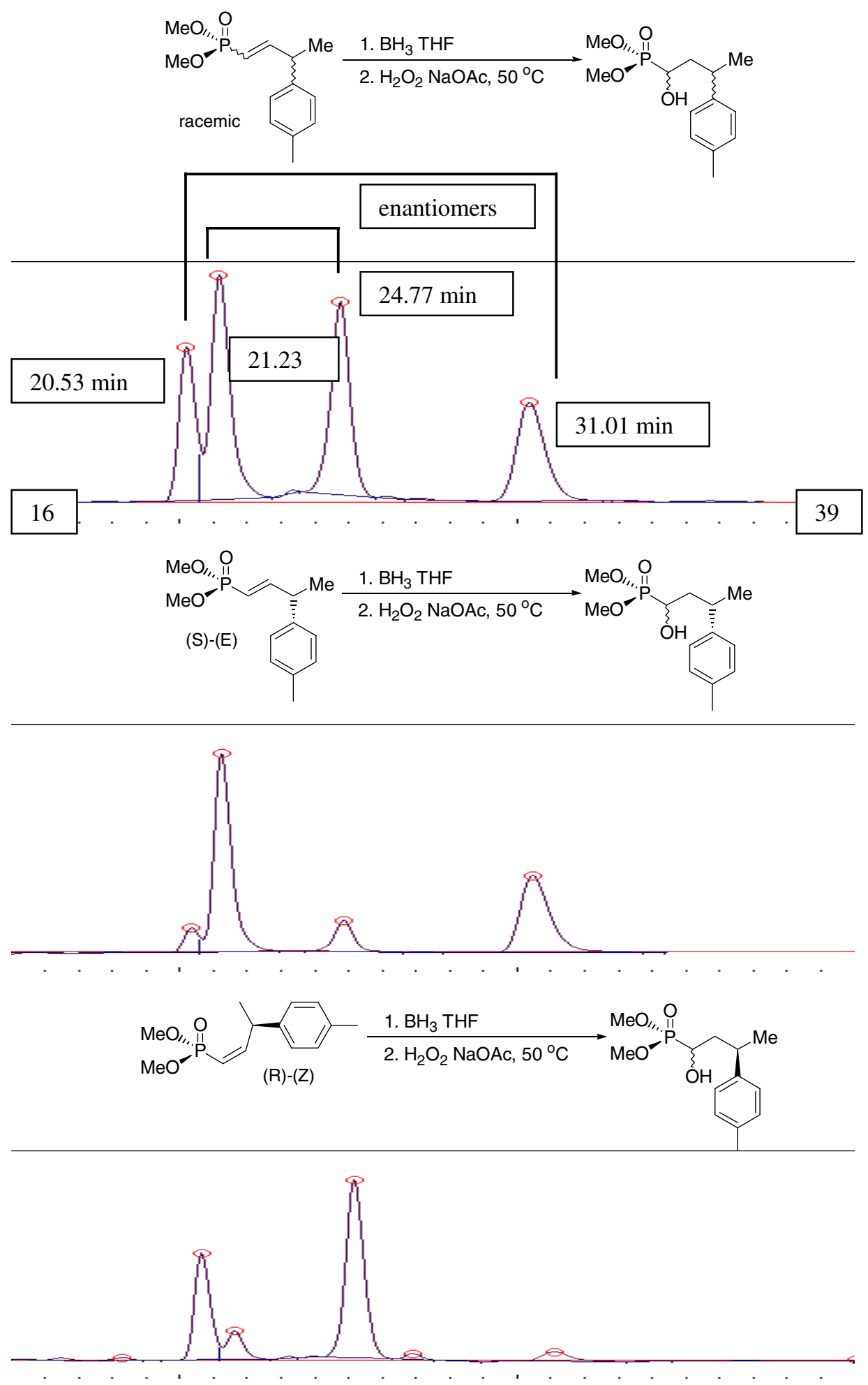